\title{
On the Growth of Iterated Monodromy Groups
}

\author{
Kai-Uwe Bux Rodrigo Pérez*
}

May 17, 2004

The iteration of a quadratic polynomial $f=f_{c}(z)=z^{2}+c$ describes a dynamical system in $\overline{\mathbb{C}}$. The behavior of this system is ruled by the geometry of the orbit

$$
\mathcal{O}=\mathcal{O}_{c}:=\left\{f(0), f(f(0)), \ldots, f^{i}(0), \ldots\right\}
$$

of its unique critical point [Miln99]. V. Nekrashevych [Nekr03] associates to each such system a group of automorphisms of the infinite binary rooted tree $T^{(2)}$. In Section 1 , we will sketch the construction of this group known as the iterated monodromy group of $f$ denoted by IMG $(f)$. This note addresses the following conjecture:

Conjecture 1 (Nekrashevych). Suppose the critical orbit of $f$ is postcritically finite, i.e., the orbit of the critical point 0 is finite and does not contain 0. Then IMG $(f)$ has intermediate growth.

We want to illustrate some of the difficulties that arise in attacking this conjecture. Our plan is to present three examples $\mathcal{G}, \mathcal{H}$ and $\mathcal{I}$ of finitely generated subgroups of $\operatorname{Aut}\left(T^{(2)}\right)$, all of which have subexponential growth. The group $\mathcal{G}$ is the First Grigorchuk group; it was the first known example of a group of intermediate growth [Grig83]. The group $\mathcal{H}$ belongs to the family of groups of intermediate growth studied in [Grig84]. Our proofs of subexponential growth for $\mathcal{G}$ and $\mathcal{H}$ are designed to illustrate the use of Proposition 10. We apply these ideas to prove subexponential growth on $\mathcal{I}:=\operatorname{IMG}\left(z^{2}+i\right)$, thus providing the first non-trivial example to support Conjecture 1.

\section{The Iterated Monodromy Group}

The postcritical set of $f$ is the closure $\overline{\mathcal{O}}$ of the critical orbit $\mathcal{O}=\left\{f^{i}(0) \mid i \geq 1\right\}$. Note that $f(\overline{\mathcal{O}}) \subseteq \overline{\mathcal{O}}$, whence $f^{-1}(\overline{\mathbb{C}} \backslash \overline{\mathcal{O}}) \subseteq \overline{\mathbb{C}} \backslash \overline{\mathcal{O}}$ and $f: f^{-1}(\overline{\mathbb{C}} \backslash \overline{\mathcal{O}}) \rightarrow \overline{\mathbb{C}} \backslash \overline{\mathcal{O}}$ is a 2 to 1 covering map.

Department of Mathematics, Cornell University, Ithaca, NY 14850.

Keywords: Iterated monodromy group, Group growth, Julia set.

2000 AMS Classification: 20F65, 37F20.

*Research supported by NSF Postdoctoral Fellowship, grant DMS-0202519. 


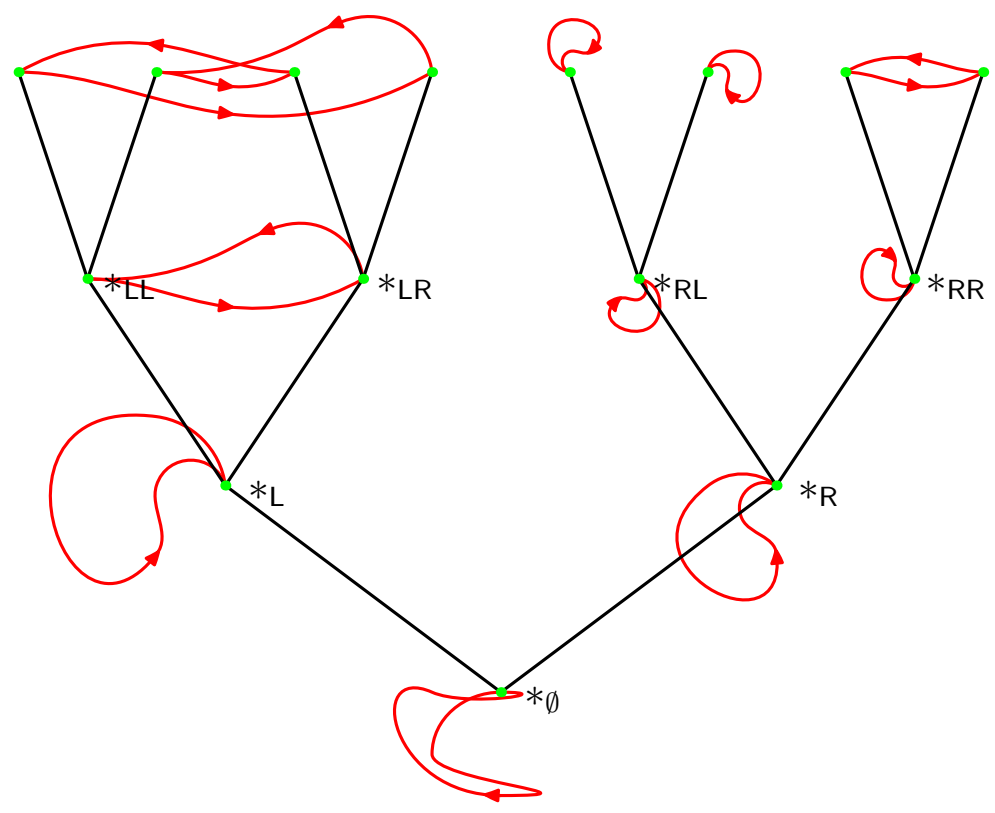

Figure 1: The monodromy action on $T^{(2)}$

Fix a base-point $*=*_{\emptyset} \in \overline{\mathbb{C}} \backslash \overline{\mathcal{O}}$. This point has two $f$-preimages $*_{\mathrm{L}}$ and $*_{\mathrm{R}}$. For any loop $\gamma$ in $\overline{\mathbb{C}} \backslash \overline{\mathcal{O}}$ based at $*$, the homotopy class $[\gamma] \in \pi_{1}(\overline{\mathbb{C}} \backslash \overline{\mathcal{O}}, *)$ determines whether $\gamma$ lifts to two loops (based at $*_{\mathrm{L}}$ and $*_{\mathrm{R}}$ ) or to two paths, one from $*_{\mathrm{L}}$ to $*_{\mathrm{R}}$ and one in the opposite direction. The monodromy action of $[\gamma] \in \pi_{1}(\overline{\mathbb{C}} \backslash \overline{\mathcal{O}}, *)$ on

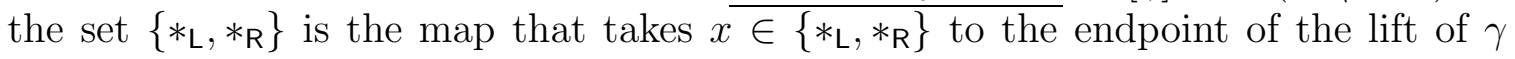
based at $x$.

Note that both $*_{\mathrm{L}}$ and $*_{\mathrm{R}}$ have two $f$-preimages: $*_{\mathrm{LL}}, *_{\mathrm{LR}}$ and $*_{\mathrm{RL}}, *_{\mathrm{RR}}$ respectively. Since $f: f^{-1}(\overline{\mathbb{C}} \backslash \overline{\mathcal{O}}) \rightarrow \overline{\mathbb{C}} \backslash \overline{\mathcal{O}}$ is a two sheeted covering map, all these four points are different. The loop $\gamma$ has four different $f^{2}$-lifts, one starting at each of the points $*_{\mathrm{LL}}, *_{\mathrm{LR}}, *_{\mathrm{RL}}, *_{\mathrm{RL}}$. Sending each of these four points to the endpoint of the corresponding $f^{2}$-lift of $\gamma$ defines an action of $\pi_{1}(\overline{\mathbb{C}} \backslash \overline{\mathcal{O}}, *)$ on the set $f^{-2}(*)=\left\{*_{\mathrm{LL}}, *_{\mathrm{LR}}, *_{\mathrm{RL}}, *_{\mathrm{RL}}\right\}$. Iterating this procedure, we define the monodromy action of $\pi_{1}(\overline{\mathbb{C}} \backslash \overline{\mathcal{O}}, *)$ on the sets $f^{-s}(*)$. Figure 1 illustrates these actions. There, we have arranged the sets $f^{-s}(*)$ into an infinite binary rooted tree. The edges from the vertices at level $s$ to their level $s-1$ parents correspond to the map $f: f^{-s}(*) \rightarrow f^{-(s-1)}(*)$.

Remark 2. Figure 1 is slightly misleading: Although we have drawn various lifts of the loop $\gamma$, the abstract tree does not lie inside $\overline{\mathbb{C}} \backslash \overline{\mathcal{O}}$. Indeed, vertices of different levels might correspond to the same point in $\overline{\mathbb{C}} \backslash \overline{\mathcal{O}}$. This occurs exactly when $*$ is a periodic point of $f$.

To avoid this ambiguity, we transfer the monodromy action to an abstract binary tree, still denoted by $T^{(2)}$, with vertices labelled by words in $\{L, R\}$ in accordance to the indices used above for the preimages of $*$.

Note that the monodromy actions of $\pi_{1}(\overline{\mathbb{C}} \backslash \overline{\mathcal{O}}, *)$ on the different levels are compatible 
with the edges in the tree $T^{(2)}$. The iterated monodromy action is the action of $\pi_{1}(\overline{\mathbb{C}} \backslash \overline{\mathcal{O}}, *)$ on $T^{(2)}$ thus induced. The iterated monodromy group of $f$ is the image

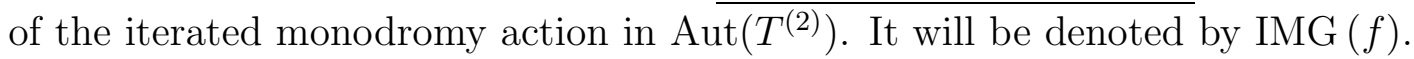

Observation 3. When indexing the iterated preimages of $*$, we make choices at each step about which preimage we call $\mathrm{L}$ and which one we call $\mathrm{R}$. Any two labellings of the iterated preimages are conjugate via an automorphism of $T^{(2)}$. Thus the iterated monodromy group is well defined up to conjugation in $\operatorname{Aut}\left(T^{(2)}\right)$.

\section{Automorphisms of the Infinite Binary Tree $T^{(2)}$}

The infinite binary rooted tree $T^{(2)}$ has two subtrees connecting to the root vertex. We call them $T_{\mathrm{L}}^{(2)}$ (left) and $T_{\mathrm{R}}^{(2)}$ (right). Both subtrees are binary infinite rooted trees in their own right. Fix isomorphisms $T_{\mathrm{L}}^{(2)} \cong T^{(2)}$ and $T_{\mathrm{R}}^{(2)} \cong T^{(2)}$. Using these identifications, any two automorphisms $\alpha_{\mathrm{L}}, \alpha_{\mathrm{R}} \in \operatorname{Aut}\left(T^{(2)}\right)$ can be combined to define an automorphism $\alpha:=\left(\alpha_{\mathrm{L}}, \alpha_{\mathrm{R}}\right) \in \operatorname{Aut}\left(T^{(2)}\right)$ : the automorphism $\alpha$ preserves each of the two subtrees and acts in $T_{\mathrm{L}}^{(2)}$ as $\alpha_{\mathrm{L}}$ and in $T_{\mathrm{R}}^{(2)}$ as $\alpha_{\mathrm{R}}$.

Moreover, the isomorphism $T_{\mathrm{L}}^{(2)} \cong T^{(2)} \cong T_{\mathrm{R}}^{(2)}$ provides a distinguished involution $\sigma \in \operatorname{Aut}\left(T^{(2)}\right)$, called the swap, that interchanges $T_{\mathrm{L}}^{(2)}$ and $T_{\mathrm{R}}^{(2)}$. Note that the swap interacts nicely with the pair notation from above:

$$
\sigma\left(\alpha_{\mathrm{L}}, \alpha_{\mathrm{R}}\right) \sigma^{-1}=\left(\alpha_{\mathrm{R}}, \alpha_{\mathrm{L}}\right)
$$

It is easy to see that this defines a wreath product decomposition

$$
\operatorname{Aut}\left(T^{(2)}\right)=\left(\operatorname{Aut}\left(T^{(2)}\right) \times \operatorname{Aut}\left(T^{(2)}\right)\right) \rtimes\langle\sigma\rangle
$$

which allows us to represent automorphisms of $T^{(2)}$ pictorially:

$$
\left.\left(\alpha_{\mathrm{L}}, \alpha_{\mathrm{R}}\right) \sigma={ }_{\sigma}^{\alpha_{\mathrm{L}}}\right\rangle^{\alpha_{\mathrm{R}}} \quad\left(\alpha_{\mathrm{L}}, \alpha_{\mathrm{R}}\right)={ }^{\alpha_{\mathrm{L}}} \searrow_{1} \gamma^{\alpha_{\mathrm{R}}}
$$

Lemma 4. For $i=1, \ldots, r$, let $x_{i}$ be variables with values in $\operatorname{Aut}\left(T^{(2)}\right)$, let $v_{i}$ and $u_{i}$ be words in $\sigma$ and the variables. For each index $i$, fix $\varepsilon_{i} \in\{1, \sigma\}$. Then the system

$$
\begin{aligned}
x_{1} & =\left(v_{1}, u_{1}\right) \varepsilon_{1} \\
& \vdots \\
x_{r} & =\left(v_{r}, u_{r}\right) \varepsilon_{r}
\end{aligned}
$$

has a unique solution.

Instead of a formal proof, we give a convincing example: 
Example 5. Consider the system

$$
\begin{aligned}
\alpha & =(\sigma, \beta) \\
\beta & =(\sigma, \gamma) \\
\gamma & =(1, \alpha)
\end{aligned}
$$

First, we obtain information about how these three automorphisms act on the first layer in $T^{(2)}$ :

$$
\alpha=\backslash_{1}{ }^{\beta} \quad \beta=\backslash_{1} \nearrow^{\gamma} \gamma=\backslash_{1} \nearrow^{\alpha}
$$

Now, we substitute and extend the picture to the second layer:

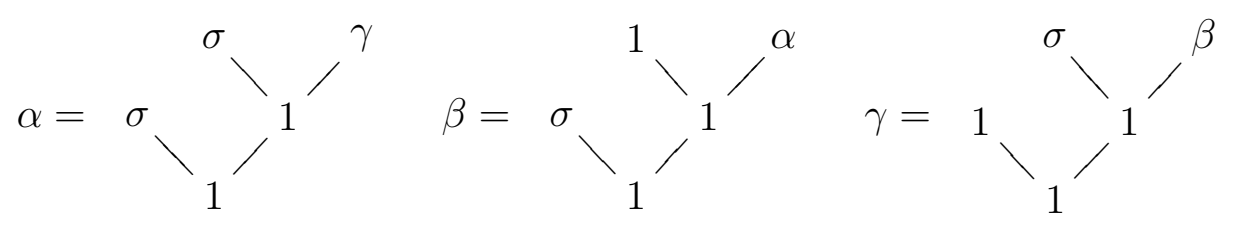

We continue and see that the system of equations determines $\alpha, \beta$, and $\gamma$ completely.

Finally, we introduce a bit of notation. For any subgroup $G \leq \operatorname{Aut}\left(T^{(2)}\right)$, we let $G_{s}$ denote the subgroup of those elements in $G$ that fix pointwise the set of vertices within distance $s$ from the root. An automorphism can be written as a pair $\left(\alpha_{\mathrm{L}}, \alpha_{\mathrm{R}}\right)$ if and only if it is in $G_{1}$. We call those elements of $G$ even. Note that $G_{s}$ is a normal subgroup of finite index in $G$. The even elements from a subgroup of index at most 2 .

\section{Group Growth via Weights}

Definition 6. Consider a group $G$ with a fixed finite generating set $\Sigma$. Any map

$$
\tilde{\ell}: \Sigma \rightarrow \mathbb{R}^{+},
$$

assigning a strictly positive weight to each generator, extends to a length function on the set $\Sigma^{*}$ of words over the alphabet $\Sigma \cup \Sigma^{-1}$ :

$$
\begin{aligned}
\tilde{\ell}: \Sigma^{*} & \rightarrow \mathbb{R}_{0}^{+} \\
w=x_{1}^{\varepsilon_{1}} x_{2}^{\varepsilon_{2}} \cdots x_{r}^{\varepsilon_{r}} & \left.\mapsto \sum_{i=1}^{r} \tilde{\ell}\left(x_{i}\right) \quad \text { (here } \varepsilon_{i}= \pm 1\right)
\end{aligned}
$$

This length descends to a length function $\ell$ on $G$ as follows:

$$
\begin{aligned}
\ell: G & \rightarrow \mathbb{R}_{0}^{+} \\
g & \mapsto \min \{\tilde{\ell}(w) \mid w \text { represents } g\}
\end{aligned}
$$


Observation 7. The map $\ell: G \rightarrow \mathbb{R}_{0}^{+}$satisfies the following conditions:

1. For any group element $g \in G$, we have $\ell(g) \geq 0$.

2. We have $\ell(g)=0$ if and only if $g=1$.

3. For any two group elements $g, h \in G$, the inequality $\ell(g h) \leq \ell(g)+\ell(h)$ holds.

4. For any radius $r$, the set $B_{\ell}(r):=\{g \in G \mid \ell(g) \leq r\}$ is finite. We call this set the ball of radius $r$.

Definition 8. The growth function $\operatorname{gr}_{\tilde{\ell}}$ associated to $\Sigma$ and $\tilde{\ell}: \Sigma \rightarrow \mathbb{R}^{+}$is defined as the "combinatorial volume" of the ball of radius $r$ :

$$
\operatorname{gr}(r)=\operatorname{gr}_{\tilde{\ell}}(r):=\#\{g \in G \mid \ell(g) \leq r\}
$$

The growth function is almost submultiplicative: There is a constant $C$ such that

$$
\operatorname{gr}\left(r+r^{\prime}\right) \leq C \operatorname{gr}(r) \operatorname{gr}\left(r^{\prime}\right)
$$

It follows that the growth rate

$$
\lambda=\lim _{r \rightarrow \infty}(\operatorname{gr}(r))^{1 / r}
$$

exists. We say that $G$ has exponential growth if $\lambda>1$ and subexponential growth if $\lambda \leq 1$.

Remark 9. The growth rate depends on the choices of $\Sigma$ and $\tilde{\ell}: \Sigma \rightarrow \mathbb{R}^{+}$. However, any other choice will yield a Lipschitz equivalent length function on $G$. It follows that whether $G$ has exponential or subexponential growth does not depend on these choices.

The following proposition is modeled upon the standard proof of subexponential growth for the First Grigorchuk group.

Proposition 10. Let $H$ be a finite index subgroup of the finitely generated group $G$, and let $\ell$ be a length function on $G$ as above. Suppose there exists numbers $\eta \in[0,1)$, $p \in(0,1], K \geq 0$, and an injective homomorphism

$$
\begin{aligned}
\phi: H & \rightarrow \overbrace{G \times \cdots \times G}^{n \text { factors }} \\
h & \mapsto\left(\phi_{1}(h), \ldots, \phi_{n}(h)\right)
\end{aligned}
$$

such that the following condition holds: 
For each $r$, the proportion of all elements in $\{h \in H \mid \ell(h) \leq r\}$ satisfying

$$
\sum_{i=1}^{n} \ell\left(\phi_{i}(h)\right) \leq \eta r+K
$$

is at least $p$.

Then $G$ has subexponential growth.

Proof. Since $H$ has finite index, there is a constant $C$ such that every $H$-coset in $G$ has a representative of length $\leq C$. Now assume, let gr be the growth function and $\lambda$ be its growth rate. Then we have, for any $\varepsilon>0$ and $r$ large enough:

$$
\begin{aligned}
(\lambda-\varepsilon)^{r} & \leq \operatorname{gr}(r) \\
& =\#\{g \in G \mid \ell(g) \leq r\} \\
& \leq \#\{h \in H \mid \ell(h) \leq r+C\} \\
& \leq \frac{1}{p} \#\left\{h \in H \mid \ell(h) \leq r+C \text { and } \sum_{i=1}^{n} \ell\left(\phi_{i}(h)\right) \leq \eta(r+C)+K\right\} \\
& \leq \frac{1}{p} \int_{\ell_{1}+\cdots+\ell_{n} \leq \eta(r+C)+K}^{\substack{\ell_{i} \geq 0 \\
\ln }} \operatorname{gr}\left(\ell_{1}\right) \cdots \operatorname{gr}\left(\ell_{n}\right) \mathrm{d} \ell_{1} \cdots \mathrm{d} \ell_{n} \\
& \leq P_{n}(r) \lambda^{\eta(r+C)+K}
\end{aligned}
$$

where $P_{n}$ is a polynomial of degree $n$. It follows that $\lambda \leq 1$ and $G$ has subexponential growth.

q.e.d.

\section{Growth in Grigorchuk's First Group $\mathcal{G}$}

The First Grigorchuk group is defined as $\mathcal{G}:=\langle\sigma, \alpha, \beta, \gamma\rangle$ where $\alpha, \beta$, and $\gamma$ are binary tree automorphisms defined by the following system:

$$
\begin{aligned}
& \alpha=(\sigma, \beta) \\
& \beta=(\sigma, \gamma) \\
& \gamma=(1, \alpha)
\end{aligned}
$$

We start by recalling some well known facts about $\mathcal{G}$.

Lemma 11. The set $\{1, \alpha, \beta, \gamma\}$ is a subgroup of $\mathcal{G}$ isomorphic to $\mathbb{Z}_{2} \times \mathbb{Z}_{2}$.

Proof. The system

$$
\begin{aligned}
& x=(1, y) \\
& y=(1, z) \\
& z=(1, x)
\end{aligned}
$$


clearly defines $x=y=z=1$. On the other hand, one easily verifies that $\left(\alpha^{2}, \beta^{2}, \gamma^{2}\right)$ and $(\alpha \beta \gamma, \beta \gamma \alpha, \gamma \alpha \beta)$ are also solutions to that system. More specifically, one obtains the following systems from the defining equations:

$$
\begin{array}{ll}
\alpha^{2}=\left(1, \beta^{2}\right) & \alpha \beta \gamma=(1, \beta \gamma \alpha) \\
\beta^{2}=\left(1, \gamma^{2}\right) & \beta \gamma \alpha=(1, \gamma \alpha \beta) \\
\gamma^{2}=\left(1, \alpha^{2}\right) & \gamma \alpha \beta=(1, \alpha \beta \gamma)
\end{array}
$$

Thus, the group generated by $\alpha, \beta$, and $\gamma$ is a quotient of $\mathbb{Z}_{2} \times \mathbb{Z}_{2}$.

Now, we see that $\alpha=(\sigma, \beta)$ acts nontrivially on the left subtree. Thus $\alpha \neq 1$. This implies $\gamma \neq 1$. Finally, $\alpha=(\sigma, \beta) \neq(1, \alpha)=\gamma$. $\quad$ q.e.d.

Historically, $\mathcal{G}$ was the first group shown to have intermediate growth [Grig83]. The proof of Theorem 12 included here is the result of several simplifications.

Theorem 12 (Grigorchuk). $\mathcal{G}$ has subexponential growth.

Proof. Even elements are represented by words containing an even number of $\sigma$ letters. As a subgroup, $\mathcal{G}_{1}$ is generated by the elements $\alpha=(\sigma, \beta), \quad \beta=(\sigma, \gamma), \quad \gamma=$ $(1, \alpha), \quad \sigma \alpha \sigma=(\beta, \sigma), \quad \sigma \beta \sigma=(\gamma, \sigma), \quad \sigma \alpha \sigma=(\alpha, 1)$. As a consequence, there is an injective homomorphism

$$
\left(\phi_{\mathrm{L}}, \phi_{\mathrm{R}}\right): \mathcal{G}_{1} \hookrightarrow \mathcal{G} \times \mathcal{G} .
$$

Consider the length function $\ell$ induced by the weights

$$
\tilde{\ell}(\sigma):=3 \quad \tilde{\ell}(\alpha):=5 \quad \tilde{\ell}(\beta):=4 \quad \tilde{\ell}(\gamma):=3 .
$$

We claim that $\left(\phi_{\mathrm{L}}, \phi_{\mathrm{R}}\right)$ and $\ell$ satisfy the hypotheses of Proposition 10 with $\eta=\frac{7}{8}$, $p=1$, and $K=3$.

It follows from Lemma 11 that every minimum length word (i.e., a word that is a minimum length representative for the group element it represents) alternates between $\sigma$ letters and the other letters. In other words a minimum length word does not contain two adjacent $\sigma$ letters (because they would cancel out) nor does it contain two adjacent non- $\sigma$ letters (because they would cancel or multiply to yield just one letter). Thus, in order to establish the hypotheses of Proposition 10, it suffices to consider a word

$$
w=(\sigma) x_{1} \sigma x_{2} \cdots \sigma x_{r}(\sigma)
$$

with an even number of $\sigma$ letters (the ones at the ends possibly being omitted) and where $x_{i} \in\{\alpha, \beta, \gamma\}$. Split $w$ into blocks of four letters, possibly followed by a single shorter block at the end. The homomorphism $\left(\phi_{\mathrm{L}}, \phi_{\mathrm{R}}\right)$ will reduce the length of each four letter block by a factor of at least $\frac{7}{8}$. For instance, $\sigma \alpha \sigma \beta \mapsto(\beta \sigma, \sigma \gamma)$ corresponds to a reduction from length 15 to 13 . The worst case is attained by the block $\sigma \alpha \sigma \alpha$ which yields a reduction from 16 to 14 . Analyzing the effect of the trailing block requires a finite amount of checking. One obtains that for any element $g \in \mathcal{G}_{1}$,

$$
\ell\left(\phi_{\mathrm{L}}(g)\right)+\ell\left(\phi_{\mathrm{R}}(g)\right) \leq \frac{7}{8} \ell(g)+3
$$

as claimed, and the result follows.

q.e.d. 
Remark 13. There is nothing magic about the weights we used, and there are other weights that would do just as well. A particularly good assignment of weights was used in [Bart00] to obtain a good explicit upper bound for the growth in $\mathcal{G}$.

\section{Growth in $\mathcal{H}$}

In this section, we will study the group $\mathcal{H}:=\langle\sigma, \alpha, \beta\rangle$ where $\alpha$ and $\beta$ are defined by the following equations:

$$
\begin{aligned}
& \alpha=(\sigma, \beta) \\
& \beta=(1, \alpha)
\end{aligned}
$$

This group serves as a model to understand the slightly more involved group $\mathcal{I}$ introduced in the next section. The group $\mathcal{H}$ is less manageable than $\mathcal{G}$ as it contains a "self-replicating element" of infinite order. For this reason, we will use the full power of Proposition 10 with a proportion factor $p$ strictly less than 1.

Lemma 14. The elements $\alpha$ and $\beta$ generate a copy of $\mathbb{Z}_{2} \times \mathbb{Z}_{2}$ inside $\mathcal{H}$.

Proof. We have:

$$
\begin{array}{ll}
\alpha^{2}=\left(1, \beta^{2}\right) & \alpha \beta \alpha \beta=(1, \beta \alpha \beta \alpha) \\
\beta^{2}=\left(1, \alpha^{2}\right) & \beta \alpha \beta \alpha=(1, \alpha \beta \alpha \beta)
\end{array}
$$

Therefore:

$$
\alpha^{2}=\beta^{2}=(\alpha \beta)^{2}=1 \text {. }
$$

Thus $\alpha$ and $\beta$ generate a quotient of $\mathbb{Z}_{2} \times \mathbb{Z}_{2}$. Since $\alpha$ acts like $\sigma$ in the left subtree, we have $\alpha \neq 1$, which in turn implies $\beta \neq 1$. Moreover, $\alpha \neq \beta$ by their actions on the left subtree. Hence $\langle\alpha, \beta\rangle$ has more than two elements.

q.e.d.

Proposition 15. The elements $\sigma \alpha$ and $\sigma \beta$ have finite order, but the element $\sigma \gamma$ has infinite order.

Proof. First, we observe that

$$
(\sigma \beta)^{4}=(\alpha, \alpha)^{2}=(1,1)=1
$$

and

$$
(\sigma \alpha)^{2}=(\beta \sigma, \sigma \beta) .
$$

Thus, $\sigma \beta$ has order 4 and $\sigma \alpha$ has order 8.

On the other hand, $\gamma$ replicates itself:

$$
(\sigma \gamma)^{2}=\sigma \alpha \beta \sigma \alpha \beta=(\beta, \sigma)(\alpha, 1)(\sigma, \beta)(1, \alpha)=(\beta \alpha \sigma, \sigma \beta \alpha)=(\gamma \sigma, \sigma \gamma) .
$$


Then

$$
(\sigma \gamma)^{2 n}=\left((\gamma \sigma)^{n},(\sigma \gamma)^{n}\right) .
$$

Therefore, $(\sigma \gamma)^{2 n}=1$ implies $(\sigma \gamma)^{n}=1$. So the order of $\sigma \gamma$ is either odd or infinite. But any odd power of $\sigma \gamma$ acts non-trivially on the tree $T^{(2)}$ as it performs a swap at the root vertex.

q.e.d.

Remark 16. The identity

$$
(\sigma \gamma)^{2 n}=\left((\gamma \sigma)^{n},(\sigma \gamma)^{n}\right)
$$

rules out any hope that one could prove subexponential growth as easily as for Grigorchuk's group $\mathcal{G}$ : A block of the form $\sigma \gamma \sigma \gamma$ inside an alternating word does not display any length reduction regardless of the choice of weights for the generators. Thus, there is no uniform reduction for all alternating words.

Having located the problem, we propose a solution.

First, we induce an appropriate length function $\ell$ on $\mathcal{H}$ via the weights

$$
\tilde{\ell}(\sigma):=3 \quad \tilde{\ell}(\alpha):=5 \quad \tilde{\ell}(\beta):=4 \quad \tilde{\ell}(\gamma):=3 .
$$

Note that minimum length words alternate between the letter $\sigma$ and the other letters.

Since there is no uniform reduction, we will introduce a measure that allows us to distinguish between group elements that have a good reduction and those that do not. Let $\varepsilon$ be a small positive parameter to be chosen later.

Definition 17. An alternating word $w$ with $n$ non- $\sigma$ letters is said to be $\varepsilon$-bad if it contains at most $\varepsilon n$ letters $\alpha$ and $\beta$. An even element $h \in \mathcal{H}_{1}$ is called $\varepsilon$-bad if every minimum length representative of $h$ is $\varepsilon$-bad. The element $h$ is $\varepsilon$-good if it is not $\varepsilon$-bad.

Note that for small $\varepsilon$, the $\varepsilon$-bad words will form only a slim fraction of all words of a given length. This, however, does not imply that the $\varepsilon$-bad group elements are a minority among the group elements of a given length: it is conceivable that all the $\varepsilon$-bad words represent different group elements whereas the $\varepsilon$-good words represent only a handful of different elements. This is the main issue to be addressed in our proof of the following:

Proposition 18 (Grigorchuk). $\mathcal{H}$ has subexponential growth.

Proof. Again, we will consider the "split into a pair" homomorphism

$$
\left(\phi_{\mathrm{L}}, \phi_{\mathrm{R}}\right): \mathcal{H}_{1} \rightarrow \mathcal{H} \times \mathcal{H}
$$

defined on the subgroup $\mathcal{H}_{1}$ of even elements. Our goal is to verify the hypotheses of Proposition 10 in this setting. 
For the length function specified above, an alternating word $w$ of length $\tilde{\ell}(w) \leq r$ contains at most $(r+3) / 6$ non- $\sigma$ letters. Let $b_{\varepsilon}(n)$ be the number of $\varepsilon$-bad alternating words that have $n$ non- $\sigma$ letters and represent an even element of $\mathcal{H}$. Then

$$
b_{\varepsilon}(n) \leq 2\left(\begin{array}{c}
n \\
\lfloor\varepsilon n\rfloor
\end{array}\right) 3^{\lfloor\varepsilon n\rfloor}
$$

where he leading 2 accounts for the choice of starting $w$ with $\sigma$ or not, the binomial coefficient counts the number of ways to allocate $\lfloor\varepsilon n\rfloor$ positions where $\alpha$ or $\beta$ can be placed, and the factor $3^{\lfloor\varepsilon n\rfloor}$ counts the ways of using the allocated positions: note that the letter $\gamma$ can be used in any of these locations.

We estimate the number $B_{\varepsilon}(r)$ of $\varepsilon$-bad group elements of length at most $r$ by

$$
B_{\varepsilon}(r) \leq \sum_{n=0}^{(r+3) / 6} b_{\varepsilon}(n) \leq \frac{(r+3)}{3}\left(\begin{array}{c}
(r+3) / 6 \\
\varepsilon(r+3) / 6\rfloor
\end{array}\right) 3^{\lfloor\varepsilon(r+3) / 6\rfloor}
$$

Now, suppose $\mathcal{H}$ has exponential growth. Then the index 2 subgroup $\mathcal{H}_{1}$ has exponential growth with respect to the restricted length $\ell_{\mathcal{H}_{1}}$. Let $\lambda>1$ be the growth rate of $\mathcal{H}_{1}$. Using Stirling's formula, we can choose $\varepsilon>0$ small enough so that $\lambda^{r}$ grows faster than $B_{\varepsilon}(r)$. In particular, for $r$ big enough, at least half of the elements in $\left\{h \in \mathcal{H}_{1} \mid \ell(h) \leq r\right\}$ are $\varepsilon$-good.

We finish the proof by observing that an $\varepsilon$-good element reduces at least by a factor of

$$
\eta:=\frac{4 \varepsilon+(2-\varepsilon) 3}{5 \varepsilon+(2-\varepsilon) 3}<1
$$

when written in the pair notation. The worst case is attained when among the non$\sigma$ letters, we have the largest possible number of $\gamma$ letters and all the remaining are $\alpha$. We have thus verified the hypotheses of Proposition 10 with $\eta<1$ and $p=1 / 2$.

q.e.d.

Remark 19. Note that in this proof, we had to fix the assumed growth rate $\lambda>1$ in order to find an $\varepsilon>0$ small enough to verify the hypotheses of Proposition 10 . This method will not allow us to deduce a bound of the form

$$
\operatorname{gr}(r) \leq \mathrm{e}^{\kappa r}
$$

with $\kappa<1$. Such a bound can be deduced in the case of Grigorchuk's group since

the reduction factor $\frac{7}{8}$ holds independently of the assumed growth rate. Details can be found in [Bart00].

\section{Growth in $\mathcal{I}$}

The critical orbit of the polynomial $z^{2}+i$ is

$$
0 \mapsto i \mapsto(-1+i) \mapsto-i \mapsto(-1+i)
$$


i.e., $z^{2}+i$ is postcritically finite. As a consequence, the iterated monodromy group $\mathcal{I}:=\operatorname{IMG}\left(z^{2}+i\right)$ is generated by three elements descended from loops around $i$, $(-1+i)$, and $-i$. More precisely, according to [BGN02], $\mathcal{I}=\langle\sigma, \alpha, \beta\rangle$ where $\alpha$, and $\beta$ are defined by the following equations:

$$
\begin{aligned}
& \alpha=(\sigma, \beta) \\
& \beta=(\alpha, 1)
\end{aligned}
$$

The generators $\alpha$ and $\beta$ correspond to loops around the periodic points $(-1+i)$ and $-i$, while $\sigma$ represents the swap on $T^{(2)}$, which is induced by the loop around the preperiodic point $i$.

The equations defining $\mathcal{I}$ are very similar to those for $\mathcal{H}$ and yet, a new complication appears: now we are facing several non-reducing elements.

Lemma 20. Any two of the generators span a finite dihedral group inside $\mathcal{I}$ :

$$
\begin{aligned}
& D_{4}(\sigma, \beta):=\langle\sigma, \beta\rangle=\left\langle\sigma, \beta \mid \sigma^{2}=\beta^{2}=(\sigma \beta)^{4}=1\right\rangle \\
& D_{8}(\sigma, \alpha):=\langle\sigma, \alpha\rangle=\left\langle\sigma, \alpha \mid \sigma^{2}=\alpha^{2}=(\sigma \alpha)^{8}=1\right\rangle \\
& D_{8}(\alpha, \beta):=\langle\alpha, \beta\rangle=\left\langle\alpha, \beta \mid \alpha^{2}=\beta^{2}=(\alpha \beta)^{8}=1\right\rangle
\end{aligned}
$$

Proof. Note that $\alpha$ is not trivial since it acts like $\sigma$ in the left subtree. Hence $\beta=(\alpha, 1)$ is not trivial either. Since $\alpha^{2}=\left(1, \beta^{2}\right)$ and $\beta^{2}=\left(\alpha^{2}, 1\right)$, we infer that $\alpha$ and $\beta$ have order 2 .

From $\sigma \beta \sigma \beta=(\alpha, \alpha)$ We infer that $\sigma \beta$ (and its inverse $\beta \sigma)$ have order 4. Hence $\sigma \alpha \sigma \alpha=(\beta \sigma, \sigma \beta)$ implies that $\sigma \alpha$ has order 8. Thus $\alpha \beta=(\sigma \alpha, \beta)$ has order 8, too.

q.e.d.

We want to ensure that every group element is represented by a minimum length word that alternates between the letter $\sigma$ and other generators. Using Lemma 20, we achieve this goal by adding the non-trivial elements of $D_{8}(\alpha, \beta)$ to the designated generating set for $\mathcal{I}$. For reference, we list the extended set of generators:

\begin{tabular}{c|c|cc|c|c} 
name & element & pair & name & element & pair \\
$\alpha_{1}$ & $\alpha$ & $(\sigma, \beta)$ & $\beta_{1}$ & $\beta$ & $(\alpha, 1)$ \\
$\alpha_{2}$ & $\alpha \beta$ & $(\sigma \alpha, \beta)$ & $\beta_{2}$ & $\beta \alpha$ & $(\alpha \sigma, \beta)$ \\
$\alpha_{3}$ & $\alpha \beta \alpha$ & $(\sigma \alpha \sigma, 1)$ & $\beta_{3}$ & $\beta \alpha \beta$ & $(\alpha \sigma \alpha, \beta)$ \\
$\alpha_{4}$ & $\alpha \beta \alpha \beta$ & $(\sigma \alpha \sigma \alpha, 1)$ & $\beta_{4}$ & $\beta \alpha \beta \alpha$ & $(\alpha \sigma \alpha \sigma, 1)$ \\
$\alpha_{5}$ & $\alpha \beta \alpha \beta \alpha$ & $(\sigma \alpha \sigma \alpha \sigma, \beta)$ & $\beta_{5}$ & $\beta \alpha \beta \alpha \beta$ & $(\alpha \sigma \alpha \sigma \alpha, 1)$ \\
$\alpha_{6}$ & $\alpha \beta \alpha \beta \alpha \beta$ & $(\sigma \alpha \sigma \alpha \sigma \alpha, \beta)$ & $\beta_{6}$ & $\beta \alpha \beta \alpha \beta \alpha$ & $(\alpha \sigma \alpha \sigma \alpha \sigma, \beta)$ \\
$\alpha_{7}$ & $\alpha \beta \alpha \beta \alpha \beta \alpha$ & $(\sigma \alpha \sigma \alpha \sigma \alpha \sigma, 1)$ & $\beta_{7}$ & $\beta \alpha \beta \alpha \beta \alpha \beta$ & $(\alpha \sigma \alpha \sigma \alpha \sigma \alpha, \beta)$ \\
$\alpha_{8}$ & $\alpha \beta \alpha \beta \alpha \beta \alpha \beta$ & $(\sigma \alpha \sigma \alpha \sigma \alpha \sigma \alpha, 1)$ & $\beta_{8}$ & $\beta \alpha \beta \alpha \beta \alpha \beta \alpha$ & $(\alpha \sigma \alpha \sigma \alpha \sigma \alpha \sigma, 1)$
\end{tabular}

Note that $\alpha_{8}=\beta_{8}$. 
Remark 21. The issue of choosing weights for the generators is delicate since we will insist that words do not increase in length when split into pair notation. We will choose weights only for $\sigma, \alpha$, and $\beta$; the weights for the redundant generators will simply be their lengths relative to the smaller generating set.

Note that the equation $\sigma \alpha \sigma \alpha=(\beta \sigma, \sigma \beta)$ imposes the restriction $\tilde{\ell}(\alpha) \geq \tilde{\ell}(\beta)$ while $\sigma \beta \alpha \beta \sigma \beta \alpha \beta=(\beta \alpha \sigma \alpha, \alpha \sigma \alpha \beta)$ requires $\tilde{\ell}(\beta) \geq \tilde{\ell}(\alpha)$. We will settle upon the weights

$$
\tilde{\ell}(\sigma)=3, \quad \tilde{\ell}(\alpha)=4, \quad \tilde{\ell}(\beta)=4
$$

and denote the induced length function by $\ell$.

As before, we will use the homomorphism

$$
\left(\phi_{\mathrm{L}}, \phi_{\mathrm{R}}\right): \mathcal{I}_{1} \rightarrow \mathcal{I} \times \mathcal{I}
$$

that splits even elements. This time, however, we will have to split elements up to three times. Note that every generator $x \in D_{8}(\alpha, \beta)$ satisfies $\ell\left(\phi_{\mathrm{L}}(x)\right)+\ell\left(\phi_{\mathrm{R}}(x)\right) \leq$ $\ell(\sigma)+\ell(x)$. This means that any length reduction secured in the process of splitting a word into a pair will persist in subsequent splittings. We call a generator $x \in$ $D_{8}(\alpha, \beta) \backslash\{1\}$ good by nature if the inequality above is strict:

$$
\ell\left(\phi_{\mathrm{L}}(x)\right)+\ell\left(\phi_{\mathrm{R}}(x)\right)<\ell(\sigma)+\ell(x) .
$$

Otherwise, $x$ is bad.

Observation 22. The only bad letters are $\alpha, \alpha_{2}, \beta_{2}$, and $\beta_{3}$. Any generator $x$ that is good by nature satisfies $\ell\left(\phi_{\mathrm{L}}(x)\right)+\ell\left(\phi_{\mathrm{R}}(x)\right) \leq \frac{29}{31}(\ell(\sigma)+\ell(x))$. The worst case is attained by $\beta_{7}$ since $\ell\left(\phi_{\mathrm{L}}\left(\beta_{7}\right)\right)+\ell\left(\phi_{\mathrm{R}}\left(\beta_{7}\right)\right)=29$ while $\ell(\sigma)+\ell\left(\beta_{7}\right)=31$. As a consequence, if the even group element $g=\left(\phi_{\mathrm{L}}(g), \phi_{\mathrm{R}}(g)\right)$ can be represented by an alternating minimum length word $w$ such that all non- $\sigma$ letters in $w$ are good by nature, then

$$
\ell\left(\phi_{\mathrm{L}}(g)\right)+\ell\left(\phi_{\mathrm{R}}(g)\right) \leq \frac{29}{31} \ell(w) .
$$

The presence of several bad generators poses a problem for the counting method employed in the previous section: The count of $\varepsilon$-bad words of a given size had a factor $3^{\lfloor\varepsilon\rfloor}$ accounting for the ways of writing letters (good or bad) at the selected positions. All other positions were filled with the bad letter $\gamma$. Now that there are four bad letters, we would have to include an additional factor of $4^{n-\lfloor\varepsilon n\rfloor}$. This clearly grows too fast.

To overcome this problem, we will follow the slogan "Some bad letters are good by position". The crucial insight is that some combinations of bad letters produce good letters after splitting.

Our method is based on the following two basic patterns: Let each occurrence of $\_$represent an arbitrary bad letter, and let $w$ be a minimum length word, not necessarily alternating. Note that $\phi_{\mathrm{R}}(\boldsymbol{s})=\beta$. 
(P.1) Every substring $\alpha \sigma_{\llcorner} \sigma \alpha$ in $w$ gives rise to the string $\sigma \beta \sigma$ in either $\phi_{\mathbf{L}}(w)$ or $\phi_{\mathrm{R}}(w)$ (depending on the position of $\left.\alpha \sigma\right\lrcorner \sigma \alpha$ within $w$ ).

(P.2) Every substring $\beta \sigma\lrcorner \sigma \beta$ in $w$ gives rise to the string $\alpha \beta \alpha$ in either $\phi_{\mathrm{L}}(w)$ or $\phi_{\mathrm{R}}(w)$. Possibly joining with adjacent letters, $\alpha \beta \alpha$ evaluates to a (good) generator $\alpha_{i}$ with $i \geq 3$ or $\beta_{j}$ with $j \geq 4$.

We now turn the basic patterns into a sufficient list of length reducing patterns. Let $w$ be an even word. The following claims are easily verified by using the patterns (P.1) and (P.2).

(B.1) If $\left.\left.w \in\{\alpha \sigma \sigma \sigma \alpha, \alpha \sigma\lrcorner \sigma \alpha_{2}, \beta_{2} \sigma\right\lrcorner \sigma \alpha, \beta_{2} \sigma \sigma \sigma \alpha_{2}\right\}$ then $\phi_{\mathbf{L}}(w)=x \sigma \beta \sigma y$ with $x, y \in$ $D_{8}(\alpha, \beta)$. Thus, $\phi_{\mathbf{L}}(w)$ contains a good generator.

(B.2) If $w \in\left\{\alpha_{2} \sigma\left\llcorner\sigma \beta_{2}, \alpha_{2} \sigma\right\lrcorner \sigma \beta_{3}, \beta_{3} \sigma\left\llcorner\sigma \beta_{2}, \beta_{3} \sigma\left\llcorner\sigma \beta_{3}\right\}\right.\right.$ then $\phi_{\mathrm{L}}(w)=x \sigma \alpha_{3} \sigma y$ with $x, y \in D_{8}(\alpha, \beta)$. Thus, $\phi_{\mathrm{L}}(w)$ contains a good generator.

(B.3) If $\left.\left.\left.\left.w \in\{\sigma\lrcorner \sigma \beta_{2} \sigma\right\lrcorner \sigma \beta_{3}, \beta_{2} \sigma\right\lrcorner \sigma \beta_{3} \sigma\right\lrcorner \sigma\right\}$ then $\phi_{\mathrm{L}}(w) \in\left\{\beta_{2} \sigma \beta_{2} \sigma \alpha, \alpha \sigma \beta_{2} \sigma \alpha_{2}\right\}$. Thus, after splitting, this realizes a pattern from (B.1).

(B.4) If $w \in\left\{\left\llcorner\sigma \alpha_{2} \sigma\right\lrcorner \sigma \alpha \sigma_{\iota},\left\llcorner\sigma \alpha \sigma_{\llcorner} \sigma \beta_{2} \sigma_{\iota}\right\}\right.$ then $\phi_{\mathrm{R}}(w) \in\left\{\beta \sigma \alpha_{2} \sigma \beta, \beta \sigma \beta_{2} \sigma \beta\right\}$, which realizes a pattern from (B.2).

Remark 23. Let us illustrate how (P.1) and (P.2) serve as the design principles for the list (B.1-B.4). The patterns in (B.1) are directly modeled upon (P.1); for instance: $\alpha \sigma_{\llcorner} \sigma \alpha_{2}=\alpha \sigma_{\llcorner} \sigma \alpha \beta$ features $\alpha \sigma_{\llcorner} \sigma \alpha$ as a subword. Similarly, (B.2) relies on (P.2).

The patterns in (B.3) descend from (P.1): The common substring $\beta_{2} \sigma \sigma \sigma \beta_{3}$ splits as follows $\left.\left.\phi_{\mathrm{L}}\left(\beta_{2} \sigma\right\lrcorner \sigma \beta_{3}\right)=\alpha \sigma\right\lrcorner \sigma \alpha$. Thus we recover (P.1). A similar computation explains how (B.4) is modeled after (P.2).

If a block of consecutive letters in a word $w$ matches one of the patterns (B.1)(B.4), it is called a good block. A letter within $w$ that is bad by nature is called good by position if it belongs to a good block. Blocks conforming to (B.1) or (B.2) yield an occurrence of a good generator in either $\phi_{\mathrm{L}}(w)$ or $\phi_{\mathrm{R}}(w)$, depending on the position of the block inside $w$. In either case, we will find a reduction in length after splitting $w$ a second time into $\left(\phi_{\mathrm{LL}}(w), \phi_{\mathrm{LR}}(w), \phi_{\mathrm{RL}}(w), \phi_{\mathrm{RR}}(w)\right)$. Similarly, blocks conforming to (B.3) or (B.4) display a length reduction at the third splitting $\left(\phi_{\mathrm{LLL}}(w), \cdots, \phi_{\mathrm{RRR}}(w)\right)$.

Lemma 24. For any number $i$ let $V_{i}$ be the set of all alternating words $w$ satisfying the following requirements:

- The number of non- $\sigma$ letters in $w$ is $i$.

- All non- $\sigma$ letters in $w$ are bad.

- The word $w$ does not contain good blocks. 
Then, there is a global bound $M$ such that $\# V_{i} \leq M$ for all $i$.

Proof. Consider a block $x \sigma_{\lrcorner} \sigma y$ in $w$. Out of all sixteen possible combinations for $x, y \in\left\{\alpha, \alpha_{2}, \beta_{2}, \beta_{3}\right\}$, the twelve listed in (B.1-B.4) give rise to good blocks, and consequently are not featured in $w$. Thus, there are only four possible patterns left that can occur in $w$, namely: $\left.\left.\left.\alpha \sigma\lrcorner \sigma \beta_{3}, \alpha_{2} \sigma\right\lrcorner \sigma \alpha_{2}, \beta_{2} \sigma\right\lrcorner \sigma \beta_{2}, \beta_{3} \sigma\right\lrcorner \sigma \alpha$. Thus, we find that besides minor disturbances near the ends of $w$, any two positions congruent modulo 8 will feature the same letter.

q.e.d.

Theorem 25. I has subexponential growth.

Proof. We will verify the hypotheses of Proposition 10 for the homomorphism

$$
\begin{aligned}
\mathcal{I}_{3} & \rightarrow \overbrace{\mathcal{I} \times \cdots \times \mathcal{I}}^{8 \text { times }} \\
g & \mapsto\left(\phi_{\mathrm{LLL}}(g), \cdots, \phi_{\mathrm{RRR}}(g)\right)
\end{aligned}
$$

For small $\varepsilon>0$, let $b_{\varepsilon}(n)$ count the number of alternating words representing elements in $\mathcal{I}_{3}$ and such that at most $\varepsilon n$ of its $n$ non- $\sigma$ letters are good, either by nature or by position. We have

$$
b_{\varepsilon}(n) \leq 2\left(\begin{array}{c}
n \\
\lfloor\varepsilon n\rfloor
\end{array}\right) 15^{\lfloor\varepsilon n\rfloor} M^{1+\lfloor\varepsilon n\rfloor}
$$

where the factors arise as follows:

- The leading 2 accounts for the choice of starting with the letter $\sigma$ or not.

- The binomial coefficient selects the positions for possible good letters.

- The power of 15 counts the ways of placing non- $\sigma$ letters at the selected positions.

- The power of $M$ accounts for the fact that the selected positions break the word into $1+\lfloor\varepsilon n\rfloor$ complementary components. These subwords do not contain good letters, i.e., they belong to the sets $V_{i}$ of Lemma 24 .

Since the longest generator $\alpha_{8}$ has length 32, a word of length $r$ has at most $(r+32) / 35$ non- $\sigma$ letters. It follows that the number $B_{\varepsilon}(r)$ of $\varepsilon$-bad elements in $\left\{g \in \mathcal{I}_{3} \mid \ell(g) \leq r\right\}$ is bounded from above by

$$
B_{\varepsilon}(r) \leq \sum_{n=0}^{(r+32) / 35} b_{\varepsilon}(n) .
$$

Assume, by contradiction, that $\mathcal{I}_{3}$ has exponential growth, with respect to the restricted length function $\ell_{\mathcal{I}_{3}}$, and let $\lambda>1$ be the corresponding growth rate. Choose $\varepsilon>0$ so that $\lambda^{r}$ grows faster than the number of $\varepsilon$-bad elements $B_{\varepsilon}(r)$.

The proof ends with the observation that good letters (by nature or by position) yield a definite reduction after splitting three times. Thus there is a number $\eta$ depending only on $\varepsilon$ satisfying the hypotheses of Proposition 10.

q.e.d. 


\section{References}

[Bart00] L. Bartholdi: The growth of Grigorchuk's group. preprint (2000) arXiv:math.GR/0012108

[BGN02] L. Bartholdi, R.I. Grigorchuk, V.Nekrashevych: From Fractal Groups to Fractal Sets. preprint (2002) arXiv:math.GR/0202001v4

[Grig83] R.I. Grigorchuk: On the Milnor problem of group growth. Dokl. Akad. Nauk. SSSR 271 (1983) p. $30-33$

[Grig84] R.I. Grigorchuk: Degrees of growth of finitely generated groups and the theory of invariant means. (Russian). Izv. Akad. Nauk SSSR Ser. Mat. 48 (1984), no. 5 p. $939-985$

[Miln99] J.W. Milnor: Dynamics in One Complex Variable. Vieweg (1999)

[Nekr03] V.Nekrashevich: Iterated Monodromy Groups. perprint (2003) arXiv:math.DS/0312306 